\title{
The Impact of Cannabis Use on the Dosage of Antipsychotic Drugs in Patients Admitted on the Psychiatric Ward at the University Hospital of the West Indies
}

\author{
P Thomas $^{1}$, M Gossell-Williams ${ }^{1}$, CA Sewell2, WD Abel ${ }^{2}$, D McGrowder ${ }^{3}, \mathrm{~J} \mathrm{Martin}^{2}$
}

\begin{abstract}
Objective: To assess the impact of cannabis use on the efficacy of antipsychotic drugs in male subjects presenting to the University Hospital of the West Indies (UHWI) with psychotic episodes.

Methods: Male subjects, 18-40 years old, admitted to the psychiatric ward of the UHWI between February 2013 and May 2013, diagnosed with schizophrenia, schizophreniform disorder and who tested positive for $\Delta^{9}$-tetrahydrocannabinol were recruited for the study. On day one, consenting subjects were assessed using the Brief Psychiatric Rating Scale (BPRS). Patients were prescribed seven days of an oral antipsychotic medication (haloperidol, chlorpromazine, risperidone, quetiapine, olanzapine). Medicated subjects were then reassessed using the BPRS on days three and seven. Statistical analysis involved the use of Student's t-test and repeated measure analysis of variance.

Results: In total, 20 subjects were recruited (mean age $=26.00 \pm 5.96$ years). Subjects were grouped based on the daily chlorpromazine equivalent (CPZE) dose given on day one into CPZE1 (CPZE dose of 100-300 $\mathrm{mg} ; n=8$ ) and CPZE2 (CPZE dose of 400-1250 mg; $n=12$ ). There was no significant difference in the total BPRS score between the groups on day one (CPZE1 $=41.38 \pm 16.47$ versus $C P Z E 2=49.42 \pm 25.58 ; \mathrm{p}=0.44)$; similar findings were obtained for the positive $(26.75 \pm 9.27$ versus $31.83 \pm 17.30 ; \mathrm{p}=0.46)$ and negative $(14.63 \pm 7.73$ versus $17.58 \pm 9.74 ; \mathrm{p}=0.48)$ symptom component on the BPRS. For subjects in CPZE1, there was no significant decrease in total BPRS score $\left[F_{(2,21)}=\right.$ 0.07, $\mathrm{p}=0.93]$ over the study period. For CPZE2, significant reduction in total BPRS scores was achieved $\left[F_{(2,33)}=7.12, \mathrm{p}=0.01\right]$, contributed by significant decrease in the positive $\left[F_{(2,33)}=5.64, \mathrm{p}\right.$ $=0.02)$ and negative $\left[F_{(2,33)}=7.53, \mathrm{p}=0.01\right)$ symptom components of the BPRS.

Conclusion: The findings of this study purport that male cannabis users presenting with psychotic disorders may not achieve optimal therapeutic benefit within seven days with CPZE doses $\leq 300 \mathrm{mg}$. As such, it appears that initiating treatment with CPZE doses of $>300 \mathrm{mg}$ will provide better therapeutic outcomes for this patient population.
\end{abstract}

Keywords: Antipsychotics, cannabis, chlorpromazine equivalent dose

WIMJ Open 2015; 2 (1): 23

\section{INTRODUCTION}

Significant advances have been made in the pharmacological management of psychiatric illnesses. Unfortunately, the positive impact of these advances has been at times affected by poor medication compliance, as well as genetic variability as it relates to the metabolism of psychotropic agents (1-3). This is a worrisome fact, for once a diagnosis of a psychotic illness has been made, use of antipsychotic medications is an eminent and almost unavoidable factor, unless contraindicated $(4,5)$.

There is a high prevalence rate of cannabis use amongst Jamaican males, many of whom have been

From: ${ }^{1}$ Department of Basic Medical Sciences, Section of Pharmacology, ${ }^{2}$ Department of Community Health and Psychiatry and ${ }^{3}$ Department of Pathology, The University of the West Indies, Kingston 7, Jamaica, West Indies.

Correspondence: Dr M Gossell-Williams, Department of Basic Medical Sciences, Section of Pharmacology, The University of the West Indies, Kingston 7, Jamaica, West Indies. E-mail: maxine.gossell@uwimona.edu.jm diagnosed with a psychotic disorder and are receiving prescription medication for the same. A trawl of the literature indicated that information on the direct pharmacological interactions between cannabis and antipsychotic medications seem to be undocumented. One known fact, however, is that there are higher risks of exaggerated symptoms and/or relapse in patients diagnosed with psychosis who also have a history of concomitant cannabis use. However, the underlying pathophysiology is unclear $(6,7)$.

Anecdotally, it has been reported that subjects admitted to the psychiatric ward of the University Hospital of the West Indies (UHWI) with a history of cannabis use require a higher dose of antipsychotic medication in order to achieve adequate clinical control. The aims of this study were to ascertain if the doses of antipsychotic medications prescribed by the clinicians were determined by the severity of the patients' conditions and to assess the influence of cannabis use on the efficacy of antipsychotic drug therapy. 


\section{SUBJECTS AND METHODS}

A cross-sectional study was performed at the UHWI, Department of Psychiatry, from February 2013 to May 2013, and involved male subjects between 18 and 40 years old presenting with schizophrenia or schizophreniform disorder. Consequent to the diagnosis, subjects were prescribed the requisite antipsychotic medication. Subjects receiving any concomitant medication other than an antipsychotic were excluded from the study. The protocol was approved by the University Hospital of the West Indies/University of the West Indies/Faculty of Medical Sciences Ethics Committee, and the relatives/guardians of subjects gave written informed consents for their participation.

\section{Clinical assessment}

Clinical presentation was assessed using the Brief Psychiatric Rating Scale (BPRS)-expanded version described by Dingemans et al (8) and Ventura et al $(9,10)$. This version evaluates 24 symptoms, subdivided into positive, negative, manic and depression/anxiety symptoms. Each symptom is rated on a seven-point severity scale, with no symptomatology rated as 1 and extremely severe rated as 7, and the scores are added to give a total score ranging from 24-168.

\section{Protocol}

Consented subjects were recruited for the study on day one of admission to the psychiatric ward. Urine was collected to group subjects as users and non-users of cannabis, using a SD-Bioline kit which tests for the main active metabolite of cannabis, $\Delta^{9}$-tetrahydrocannabinol (THC), giving a positive result when THC is greater than $50 \mathrm{ng} / \mathrm{ml}$. The mental health professional attached to the study was blinded from the cannabis result. Baseline clinical assessment also included measurements of body weight, height, blood pressure, waist circumference, hepatic and renal function test. Age, living status and history of psychosis were also recorded. Each subject was included in the study for seven days.

Subjects were assessed using the BPRS-expanded version form, by the trained mental health professional, upon admission to the psychiatric ward (day one). All subjects received an initial treatment with haloperidol $(10 \mathrm{mg}$ intramuscularly) upon entry into the Accident and Emergency Department. Each subject was then orally medicated with an oral antipsychotic medication (haloperidol, chlorpromazine, risperidone, quetiapine, olanzapine) and was maintained on this initial medication for seven days with a dose increase as deemed necessary by the trained mental health professional. Most of the subjects in the study $(\mathrm{n}=13,65 \%)$ were prescribed an atypical antipsychotic medication (risperidone, quetiapine, olanzapine) while the remaining subjects ( $\mathrm{n}=7$, $35 \%$ ) were prescribed haloperidol, a typical antipsychotic medication. Using chlorpromazine equivalent (CPZE) conversion charts developed by Scott Woods in 2003 (11, 12), subjects were subdivided into two groups: CPZE1 (daily
CPZE dose of 100-300 mg; $\mathrm{n}=8$ ) and CPZE2 (daily CPZE dose of 400-1250 mg; $n=12$ ). Subjects were reassessed using the BPRS on days three and seven for changes in BPRS. No other psychotropic medication was prescribed, with the exception of anticholinergics (cogentin) and/or antihistamines (diphenhydramine) for the alleviation of extrapyramidal symptoms. Subjects were also allowed to receive fluphenazine decanoate intramuscularly if needed.

\section{Data analysis}

Daily dosages of antipsychotic medications were converted to CPZE using drug comparison charts developed by Woods $(11,12)$. Response rate was calculated as (pre-treatment BPRS score minus post-treatment BPRS score/pre-treatment BPRS score) $\times 100$ and clinical improvement was measured as a response rate of $\geq 20 \%$ (13). Statistical analysis was conducted using Statistical Package for the Social Sciences (SPSS) version 20.0 for Windows with the level of significance set at $p<0.05$.

\section{RESULTS}

In total, 24 subjects were recruited; however, four were excluded from analysis as their symptomatology necessitated drug regime changes outside of the study criteria. Of the 20 male subjects completing the study, the mean $( \pm \mathrm{SD})$ age was $26.00 \pm 5.96$ years. The majority of the subjects were single $(\mathrm{n}=19,95 \%)$, received a secondary school level education ( $\mathrm{n}$ $=13,65 \%$ ), were diagnosed with schizophrenia-paranoid type $(\mathrm{n}=17,85 \%)$ and used cannabis within one week of admission $(n=13,65 \%)$ to the psychiatric ward. The demographic data for these patients are given in Table 1.

There was no significant difference in blood pressure, weight, height and abdominal circumference between the two groups. No derangement was observed in the renal function as the mean serum urea and creatinine concentrations were within the normal reference intervals of $2.5-6.7 \mathrm{mmol} / \mathrm{L}$ and 9-124 $\mu \mathrm{mol} / \mathrm{L}$, respectively. However, as it relates to the hepatic function, the mean serum aspartate aminotransferase (AST) activity was approximately two times above the upper limit of the normal reference interval of 7-32 U/L. Additionally, the serum creatine phosphokinase (CPK) activity was approximately 9.5 times above the upper reference limit, as the normal reference interval for males is 40-240 U/L (Table 1). Statistical analysis of the AST and CPK enzymes at baseline showed no significant difference between CPZE1 and CPZE2.

Table 2 shows the antipsychotics given to each subject in the study and the CPZE conversions. Twelve subjects received CPZE2 and eight received CPZE1. For the CPZE1 group, there was no significant difference in the CPZE dose from day one to day seven. For the CPZE2 group, the finding was the same, although the patient receiving haloperidol had a reduction in dose from day one. 
Table 1: Baseline (day one) demographic information of patients enrolled in the study

\begin{tabular}{|c|c|}
\hline Demographic data & Total $(n=20)$ \\
\hline $\begin{array}{l}\text { Schizophrenia, } \mathrm{n}(\%) \\
\text { Schizophreniform disorder }\end{array}$ & $\begin{array}{l}\text { Paranoid type }=17(85 \%) ; \text { Disorganized type }=1(5 \%) \\
2(10 \%)\end{array}$ \\
\hline Age, years $($ mean $\pm \mathrm{SD})$ & $26.00 \pm 5.96$ \\
\hline Ethnicity & Afro-Caribbean \\
\hline Marital status, $\mathrm{n}(\%)$ & Single $=19(95 \%) ;$ Married $=1(5 \%)$ \\
\hline Highest education level, n (\%) & Primary $=2(10 \%) ;$ Secondary $=13(65 \%) ;$ Tertiary $=5(25 \%)$ \\
\hline Occupation, n (\%) & Employed $=7(35) ;$ Unemployed $=8(40 \%) ;$ Student $=5(25 \%)$ \\
\hline Age of first use of cannabis, years (mean $\pm \mathrm{SD}$ ) & $14.23 \pm 3.17$ \\
\hline Age of first psychotic episode, years $($ mean \pm SD) & $22.20 \pm 4.16$ \\
\hline $\begin{array}{l}\text { Living status, } \mathrm{n}(\%) \\
\text { Body weight, } \mathrm{kg}(\text { mean } \pm \mathrm{SD}) \\
\text { Height, cm (mean } \pm \mathrm{SD}) \\
\text { Waist circumference, } \mathrm{cm}(\text { mean } \pm \mathrm{SD}) \\
\text { Blood pressure, } \mathrm{mmHg}(\text { mean } \pm \mathrm{SD})\end{array}$ & $\begin{array}{l}\text { Alone }=6(30 \%) ; \text { With family }=13(65 \%) ; \text { Other }=1(5 \%) \\
66.76 \pm 13.32 \\
171.71 \pm 7.16 \\
77.78 \pm 8.96 \\
\text { Systolic pressure: } 119.05 \pm 19.12 \\
\text { Diastolic pressure: } 74.25 \pm 8.12\end{array}$ \\
\hline Direct bilirubin $(\mathrm{mmol} / \mathrm{L})$ & $5.44 \pm 2.92$ \\
\hline Total bilirubin (mmol/L) & $14.67 \pm 6.95$ \\
\hline Alkaline phosphatase (U/L) & $59.06 \pm 15.32$ \\
\hline Gamma glutamyl transferase (U/L) & $26.85 \pm 10.95$ \\
\hline Aspartate aminotransferase (U/L) & $72.08 \pm 69.13$ \\
\hline Creatine phosphokinase (U/L) & $2279.89 \pm 1289.24$ \\
\hline Lactate dehydrogenase (U/L) & $302.50 \pm 151.57$ \\
\hline Albumin $(\mathrm{g} / \mathrm{L})$ & $42.53 \pm 3.56$ \\
\hline Sodium $(\mathrm{mmol} / \mathrm{L})$ & $138.06 \pm 2.11$ \\
\hline Potassium $(\mathrm{mmol} / \mathrm{L})$ & $4.05 \pm 1.14$ \\
\hline Chloride (mmol/L) & $103.35 \pm 3.69$ \\
\hline Calcium $(\mathrm{mmol} / \mathrm{L})$ & $2.37 \pm 0.09$ \\
\hline Phosphorus (mmol/L) & $1.39 \pm 1.35$ \\
\hline $\mathrm{HCO}_{3}(\mathrm{mmol} / \mathrm{L})$ & $20.08 \pm 2.50$ \\
\hline Urea (mmol/L) & $4.00 \pm 1.11$ \\
\hline Creatinine $(\mathrm{mmol} / \mathrm{L})$ & $81.06 \pm 17.79$ \\
\hline Total protein $(\mathrm{g} / \mathrm{L})$ & $72.76 \pm 4.45$ \\
\hline Total globulins $(\mathrm{g} / \mathrm{L})$ & $48.06 \pm 70.30$ \\
\hline
\end{tabular}

Table 2: Comparison of chlorpromazine equivalent (CPZE) doses between dosing groups (CPZE1, CPZE2) of prescribed antipsychotic agents on assessment days

\begin{tabular}{llll}
\hline $\begin{array}{l}\text { Antipsychotic drug } \\
\text { CPZE dose/mg }\end{array}$ & Day $\mathbf{m}$ & Day $\mathbf{3}$ & Day 7 \\
\hline $\begin{array}{l}\text { CPZE1 (n = 8) } \\
\text { Risperidone }\end{array}$ & $5.25 \pm 1.49$ & $5.25 \pm 1.49$ & $5.25 \pm 1.49$ \\
Total CPZE dose & $262.50 \pm 74.40$ & $262.50 \pm 74.40$ & $262.50 \pm 74.40$ \\
CPZE2 (n = 12) & & & \\
Haloperidol (7) & $18.57 \pm 4.76$ & $17.86 \pm 2.67$ & $17.86 \pm 2.67$ \\
& $928.57 \pm 133.63$ & $892.85 \pm 133.63$ & $892.85 \pm 133.63^{*}$ \\
Quetiapine (1) & 300 & 300 & 600 \\
& 400 & 400 & 800 \\
Olanzapine (4) & $22.50 \pm 5.00$ & 20 & 20 \\
& $450.00 \pm 100.00$ & 400 & 400 \\
Total CPZE dose & $725.00 \pm 311.52$ & $687.50 \pm 272.30$ & $720.83 \pm 258.02$ \\
\hline
\end{tabular}

*represents statistically significant decrease in the haloperidol CPZE dose over the study period $(p=0.03)$ 
The Figure shows that there was no significant difference in the total BPRS score between the groups $(\mathrm{CPZE} 1=41.38 \pm 16.47$ versus $\mathrm{CPZE} 2=49.42 \pm 25.58 ; p=$ $0.44)$; similar findings were obtained for the positive (26.75 \pm 9.27 versus $31.83 \pm 17.30 ; p=0.46)$ and negative $(14.63 \pm$ 7.73 versus $17.58 \pm 9.74 ; p=0.48)$ symptom components of the BPRS.

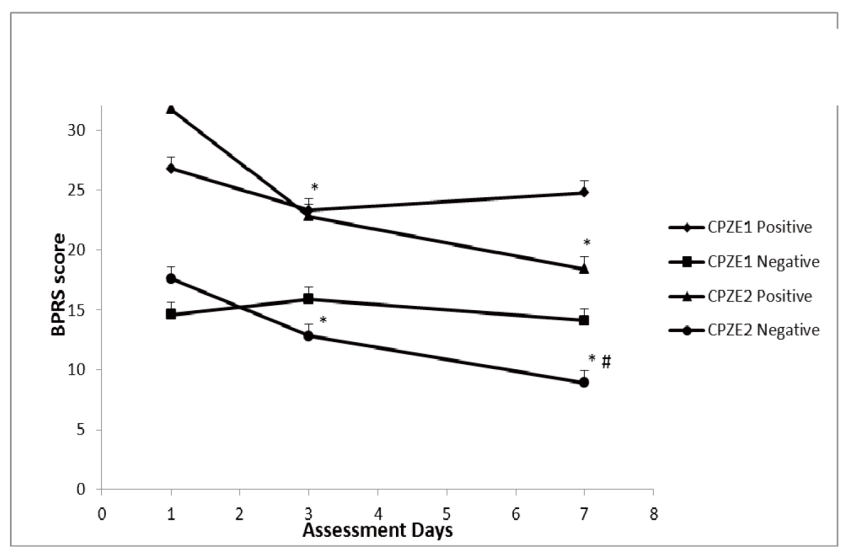

Figure: Positive and negative symptoms of the Brief Psychiatric Rating Scale (BPRS) measured on days one, three and seven of the study with chlorpromazine equivalent dosing groups (CPZE1 and CPZE2) dose ranges prescribed in the ward.

*represents significant difference in the days three and seven mean BPRS scores compared to the day one scores for the CPZE2 dose range.

\# represents the statistical difference seen in the day three versus day seven BPRS scores.

For CPZE1, the day three mean BPRS score was 39.13 \pm 10.86 and the day seven mean BPRS score was $38.88 \pm$ 15.16 , however, the change was not significantly different from day one $\left[\mathrm{F}_{(2,21)}=0.07, p=0.93\right]$. Similar findings were obtained for the positive $\left[\mathrm{F}_{(2,21)}=0.514, p=0.62\right]$ and negative $\left[\mathrm{F}_{(2,21)}=0.66, p=0.55\right]$ symptom components of the BPRS for this group.

For CPZE2, the significant decrease by day three to $35.50 \pm 18.68(p=0.01)$ through day seven to $27.33 \pm 14.88$ $(p=0.002)$ contributed to a significant decrease in mean BPRS score across the study period $\left[\mathrm{F}_{(2,33)}=7.12, p=0.01\right]$. Further assessment of the change in total BPRS score showed that the improvement in the score was contributed by a significant decrease in the positive symptom component of the BPRS $\left[\mathrm{F}_{(2,33)}=5.64, p=0.02\right]$, attributed to a significant decrease from day one to day three $(p=0.036)$ through to day seven $(p=0.005)$. There was also a significant decrease in the negative symptom components of the $\operatorname{BPRS}\left[\mathrm{F}_{(2,33)}=\right.$ $7.53, p=0.01]$, attributed to significant change from day one to day three $(p=0.012)$ through to day seven $[p=0.002]$ (Figure).

Using a change in total BPRS of $\geq 20 \%$ as the measure of clinical improvement, by day three, $25 \%(2 / 8)$ of subjects receiving CPZE1 showed a clinical improvement to the medication compared to $38.5 \%$ (5/12) receiving CPZE2. These proportions were not significantly different (Fisher's exact $p=0.642$ ). However, by day seven, while clinical improvement remained at only $25 \%$ in the CPZE1 group, clinical improvement was seen in the majority, $83.3 \%$ $(10 / 12)$, of subjects for the CPZE2 group (Fisher's exact $p=$ $0.015)$.

\section{DISCUSSION}

In this study, the BPRS was the instrument used to determine the severity of the psychotic symptoms being experienced by the subject as well as the efficacy of the prescribed treatment regime. The highest BPRS scores were obtained on day one, with a gradual decrease in the BPRS scores recorded over the study period. These findings indicated that irrespective of the type of antipsychotic drug (haloperidol, quetiapine, olanzapine, risperidone), the prescribed treatment regime was effective.

Biochemical analysis revealed an elevation in the hepatic enzymes AST and CPK activity upon admission to the study (day one). This finding is consistent with documented studies, as these enzymes are reportedly high during the acute stages of psychosis (14-19). Statistical results showed that this elevation was not significantly different between the dosing groups (CPZE1 and CPZE2).

After conversion of the prescribed medications to the relevant chlorpromazine equivalents, subjects were grouped into those receiving $100-300 \mathrm{mg}$ and those receiving 400 $1250 \mathrm{mg}$ (20). No significant difference was noted in the total BPRS scores between the dose ranges on day one. The subjects prescribed CPZE2 had significantly better outcomes on days three and seven follow-up than subjects prescribed CPZE1. Even though, from the findings, CPZE doses $\leq 300$ mg may be the minimal effective doses in patients (21), it appears that this dose is suboptimal and caution should be taken when prescribing doses $<300 \mathrm{mg}$ to male cannabis users with a diagnosis of schizophrenia or schizophreniform disorder. Clinical assessment of the subjects further underscored this finding as most of the subjects who received $>300 \mathrm{mg}$ CPZE attained clinical improvement by day seven compared to only two of eight subjects showing clinical improvement with the lower CPZE dose.

This study provides preliminary evidence that cannabis users diagnosed with a psychotic disorder may require larger doses of medication to effect eradication of symptoms compared to non-cannabis users diagnosed with a psychotic disorder. The findings are limited by the small sample size and absence of a cannabis naïve group in the study (THC < $50 \mathrm{ng} / \mathrm{ml}$ on presentation). Another limitation includes possible inter-rater variability as different mental health professionals were involved in the scoring of symptoms on the BPRS over the assessment days. It is, however, clear that the findings provide a direct link to the doses of drugs prescribed to patients with schizophrenia and schizophreniform disorder and the BPRS scores measured over a sevenday period. 
In conclusion, doses of medication prescribed to patients are directly related to the control of the severity of the psychotic impairments. In patients exposed to cannabis use, the recommended starting dose should be $>300 \mathrm{mg}$ CPZE to provide better therapeutic outcomes.

\section{REFERENCES}

1. Hasnain M, Vieweg WV, Fredrickson SK, Beatty-Brooks M, Fernandez A, Pandurangi AK. Clinical monitoring and management of the metabolic syndrome in patients receiving atypical antipsychotic medications. Primary Care Diabetes 2009; 3: 5-15.

2. Mitchell PB. Therapeutic drug monitoring of psychotropic medications. Br J Clin Pharmacol 2000; 49: 303-12.

3. Naber D, Riedel M, Klimke A, Vorbach E, Lambert M, Kuhn K et al. Randomized double blind comparison of olanzapine vs. clozapine on subjective well-being and clinical outcome in patients with schizophrenia. Acta Psychiatrica Scandinavica 2005; 111: 106-15.

4. Kerna V, Nosalova G, Ondrejka I. Metabolic risk in selected secondgeneration antipsychotics. Bratislavskè Lekàrske Listy 2010; 111: 640.

5. Uchida H, Suzuki T, Takeuchi H, Arenovich T, Mamo DC. Low dose vs standard dose of antipsychotics for relapse prevention in schizophrenia: meta-analysis. Schizophr Bull 2011; 37: 788-99.

6. Schofield D, Tennant C, Nash L, Degenhardt L, Cornish A, Hobbs C et al. Reasons for cannabis use in psychosis. Aust N Z J Psychiatry 2006; 40: $570-4$.

7. Arseneault L, Cannon M, Witton J, Murray RM. Causal association between cannabis and psychosis: examination of the evidence. $\mathrm{Br} \mathrm{J}$ Psychiatry 2004; 184: 110-7.

8. Dingemans PMAJ, Linszen DH, Lenior ME, Smeets RMW. Component structure of the expanded brief psychiatric rating scale (BPRS-E). Psychopharmacology 1995; 122: 263-7.

9. Ventura J, Green MF, Shaner A, Liberman RP. Training and quality assurance with the Brief Psychiatric Rating Scale: "the drift busters". Int J Methods Psychiatr Res 1993; 3: 221-4.

10. Ventura J, Nuechterlein KH, Subotnik KL, Gutkind D, Gilbert EA. Symptom dimensions in recent-onset schizophrenia and mania: a principal components analysis of the 24-item Brief Psychiatric Rating Scale. Psychiatry Res 2000; 97: 129-35.

11. Danivas V, Venkatasubramanian G. Current perspectives on chlorpromazine equivalents: comparing apples and oranges! Indian J Psychiatry 2013; 55: 207.

12. Woods SW. Chlorpromazine equivalent doses for the newer atypical antipsychotics. J Clin Psychiatry 2003; 64: 663-7.
13. Volonteri LS, Cerveri G, De Gaspari IF, Baldi ML, Rolandi ML, Papa P et al. Long-acting injectable risperidone and metabolic ratio: a possible index of clinical outcome in treatment-resistant schizophrenic patients. Psychopharmacology 2010; 210: 489-97.

14. Amorim M, Moreira A, Condeco J, Monteiro P, Marques AJ, Summavielle T. P-1201-Antipsychotic therapy and biochemical laboratory profile characterization of a sample of patients diagnosed with schizophrenia. European Psychiatry 2012; 27 (Suppl 1): 1.

15. Teixeira J, Rebelo D, Simões do Couto F, Figueira ML. P03-201 Requisition of blood analysis for patients with schizophrenia upon acute admission. European Psychiatry 2009; 24 (Suppl 1): S1200.

16. Garcia-Unzueta MT, Herran A, Sierra-Biddle D, Amado JA, VàzquezBarquero JL, Álvarez C. Alterations of liver function test in patients treated with antipsychotics. J Clin Lab Anal 2003; 17: 216-8.

17. Manor I, Hermesh H, Valevski A, Benjamin Y, Munitz H, Weizman A. Recurrence pattern of serum creatine phosphokinase levels in repeated acute psychosis. Biol Psychiatry 1998; 43: 288-92.

18. Meltzer HY, Ross-Stanton J. Mean serum creatine kinase activity in patients with functional psychoses. Arch Gen Psychiatry 1980; 37: 650.

19. Meltzer Y. Serum creatine phosphokinase in schizophrenia. Am J Psychiatry 1976; 33: 2.

20. Owen RR, Thrush CR, Kirchner JE, Fischer EP, Booth BM. Performance measurement for schizophrenia: adherence to guidelines for antipsychotic dose. Int J Qual Health Care 2000; 12: 475-82.

21. Raggi MA, Roberto M, Cesare S, Vincenzo P. Atypical antipsychotics: pharmacokinetics, therapeutic drug monitoring and pharmacological interactions. Curr Med Chem 2004; 11: 279-96.

Submitted 13 Oct 2014

Accepted 27 Oct 2014

Published 16 Mar 2015

Online: http://www.mona.uwi.edu/wimjopen/article/1618

(C) Thomas et al 2015

This is an open access article made freely available under Creative Commons Attribution 4.0 International (CC BY 4.0). Users are free to share, copy and adapt this work as long as the copyright holder (author) is appropriately and correctly credited. See http:// creativecommons.org/licences/by/4.0/deed.en_us for more information. 